\title{
Adaptive Black Blood Fast Spin Echo for End-Systolic Rest Cardiac Imaging
}

\author{
Brice Fernandez, ${ }^{1,2,3}$ Julien Oster, ${ }^{2,3}$ Maelene Lohezic,, ${ }^{1,2,3}$ Damien Mandry, ${ }^{2,3,4}$ \\ Olivier Pietquin, ${ }^{2,3,5}$ Pierre-André Vuissoz, ${ }^{2,3}$ and Jacques Felblinger ${ }^{2,3,4 *}$
}

\begin{abstract}
Black Blood Fast Spin Echo imaging of the heart is usually performed during mid-diastolic rest. This is a direct consequence of the long inversion time required to suppress the blood signal, which is constrained by the $T_{1}$ of the blood, and of the heart rate. To overcome these constraints, and to acquire black blood images in the end-systolic rest period, a new approach is introduced aiming at adaptively predicting the best time to prepare and acquire MR signals. It is based on a RR interval prediction algorithm and on a cardiac cycle model. The proposed method was applied to $\mathbf{1 4}$ healthy volunteers and is compared to a simple alternative method using a fixed delay and to the standard black blood imaging method for imaging in the middiastolic rest period. Results show that the proposed method offers an increased robustness in terms of trigger delay error and image quality compared to the tested simple alternative. Also, it has been shown by qualitative analysis done by an experienced observer that the right ventricle, especially the thin right ventricle free wall, is better depicted with our method than with the standard mid-diastolic rest acquisition. Magn Reson Med 000:000-000, 2010. (C) 2010 Wiley-Liss, Inc.
\end{abstract}

Key words: cardiac MRI; right ventricle; end-systolic rest; Kalman filter

The Fast spin echo (FSE) sequence (1) is a key component of cardiac MR examination. It provides an accurate depiction of the heart and the mediastinum anatomy with a high signal-to-noise ratio. It also opens the possibility of good $T_{1}$ or $T_{2}$ weighting. Nulling flowing blood signal, known as black blood imaging, is achieved thanks to a double inversion recovery (DIR) pulse pair. Combining FSE with a black blood preparation (2) enables a correct depiction of the myocardium morphology. $T_{2}$-weighted imaging of the heart provides additional information to that given by contrast enhanced MRI. Therefore, the double inversion recovery FSE (DIR-FSE) has been extensively used for various cardiac pathologies $(3,4)$. This sequence has been widely used to detect intra myocardial fat infiltration often associated with arrhythmogenic right ventricular

${ }^{1}$ Global Applied Science Laboratory, GE Healthcare, Nancy, France

${ }^{2}$ IADI Laboratory, Nancy-Université, Nancy, France

${ }^{3}$ U947, INSERM, Nancy, France

${ }^{4} \mathrm{CHU}$ de Nancy, Nancy, France

${ }^{5}$ IMS Research Group, Supelec Metz Campus, Metz, France

Presented in part at the 17th Annual Meeting of ISMRM, Honolulu, HI, USA

Grant sponsors: INSERM, FEDER, and the Region Lorraine

*Correspondence to: Pr. Jacques Felblinger, PhD, IADI Lab., INSERM U947, CHU de Nancy Brabois, Rue du Morvan, 54511 Vandoeuvre-lès-Nancy, France. E-mail: j.felblinger@chu-nancy.fr

Received 27 November 2009; revised 8 June 2010; accepted 15 June 2010. DOI 10.1002/mrm.22566

Published online in Wiley Online Library (wileyonlinelibrary.com).

(C) 2010 Wiley-Liss, Inc. dysplasia/cardiomyopathy (ARVD/C) (5,6). Another potential application is the myocardial $T_{2}$ measurement used to help the detection of acute heart transplant rejection (7).

As demonstrated by Weissler et al. (8), the duration of the systole is less sensitive to heart rate variation than the diastole one. The end-systolic rest is generally considered to last around $60-80$ msec (9) against $150-200$ msec for the mid-diastolic rest (10). However, according to a recent study (11), $26 \%$ of patients have a longer rest period at endsystole than at mid-diastole, especially patients with high heart rate. Previously cited results on cardiac rest (8-12) imply that it is always possible to perform a DIR-FSE in the end-systolic rest providing natural heart cycle changes are taken into account. Indeed, in case of high heart rate, over $85 \mathrm{bpm}$ (beats per minute), performing black blood FSE in end-systolic rest is still be feasible as the cardiac rest is less sensitive to heart rate variations (13) and longer than the mid-diastolic rest. However, given the short time between the $\mathrm{R}$ wave and the end-systolic rest, as well as the long inversion time $T_{\mathrm{I}}$ recommended (12), DIR pulses have to be played out before the R-wave to perform black blood acquisitions in end-systole. Because the end-systolic rest is relatively short, the echo train has to be placed carefully in the cardiac cycle.

An end-systolic view of the heart may be of clinical interest for pathologies such as ARVD/C $(5,6)$ and acute heart transplant rejection (7). The right ventricle wall is known to be thin; the right ventricle wall may consequently be better depicted during the end-systolic rest when the myocardium is completely contracted and thicker.

The purpose of this pilot study is the implementation and the evaluation on healthy volunteers of two methods for acquiring black blood FSE in end-systolic rest. First, a fixed delay is used to position the acquisition window in the endsystolic rest of the next cardiac cycle. The second method is novel and adaptive. Based on a cardiac cycle model and Kalman filtering (14), it allows predicting the RR interval length to start DIR preparation before the R-wave. An adaptive trigger delay is created for a correct acquisition window positioning with respect to the RR interval variation. The evaluation is made by the assessment of the depiction of left and right ventricles, as well as to the error made in the echo train placement with respect to the end-systolic rest.

\section{THEORY}

\section{DIR-FSE and Rest Periods}

Black blood fast spin echo sequences use a pair of inversion pulses. DIR preparation allows the homogeneous 


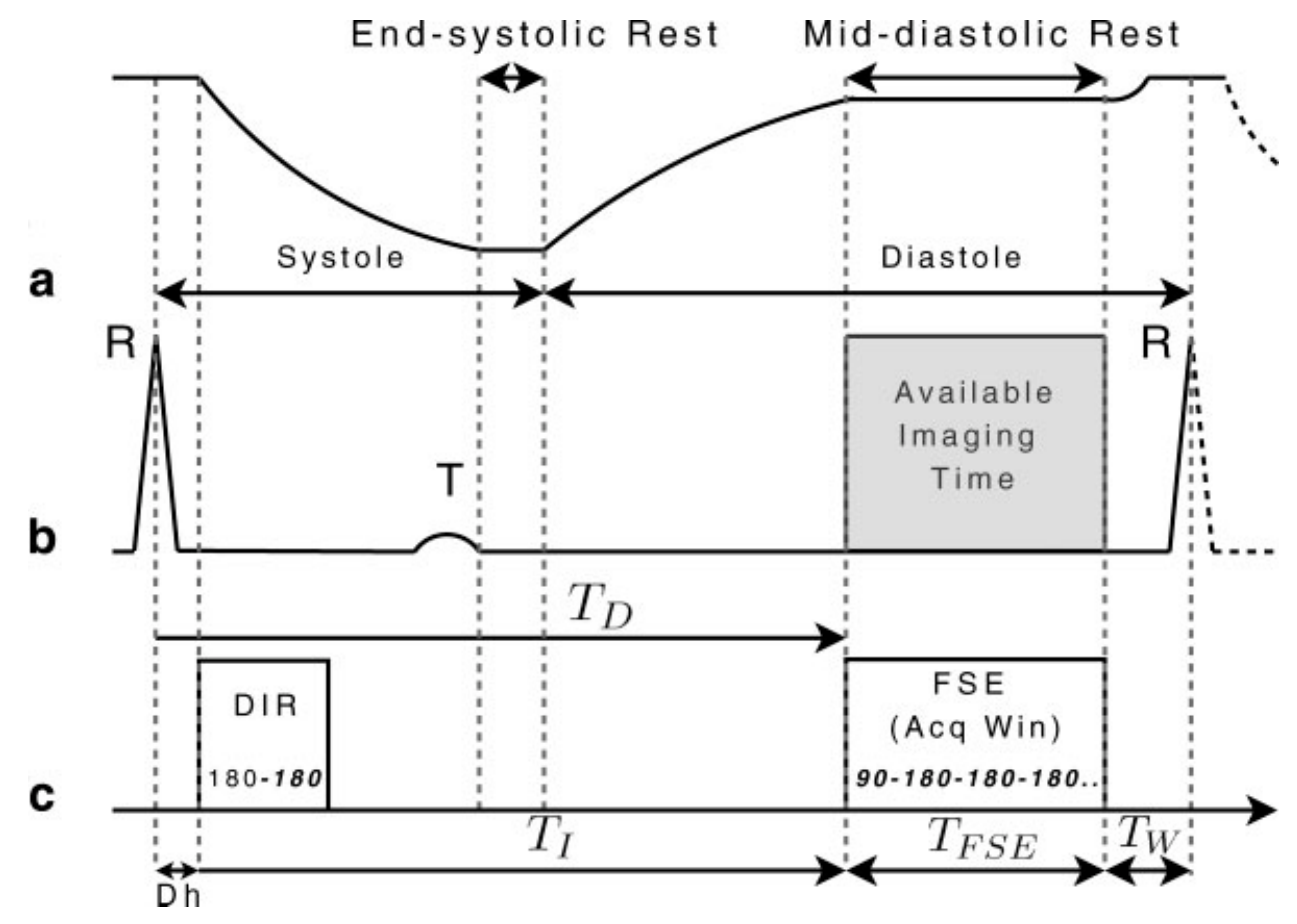

FIG. 1. Standard double inversion recovery (DIR) fast spin echo (FSE) pulse sequence with representation of the cardiac activity. a: Schematic representation of the volume of the right ventricle. Systolic and diastolic rests are noted to show the approximate length of each one compared to electrocardiogram (ECG). b: Schematic ECG with the R and the T-wave. The end-systolic rest begins just after the T-wave and the available imaging time is depicted in diastolic rest period. c: Standard DIR-FSE method. RF pulses in bold face are slice selective, $T_{\mathrm{D}}$ is the trigger delay defined as the time between the R wave and the beginning of the FSE sequence or acquisition window and $T_{1}$ is the inversion time. $T_{\mathrm{W}}$ is the trigger window defined as a dead time at the end of the cardiac cycle when the start of a new DIR-FSE sequence is inhibited. The delay $\mathrm{Dh}$ represents the time between the $\mathrm{R}$ wave and the beginning of DIR pulses that depends on specific hardware.

suppression of the blood signal except for stationary flows $(15,16)$. The optimal $T_{\mathrm{I}}$ to suppress the blood signal, $T_{\mathrm{I}, \mathrm{opt}}$, can be calculated knowing its $T_{1, \text { blood }}$ and the repetition time $T_{\mathrm{R}}$ with the well-known Fleckenstein's formula (17):

$$
T_{\mathrm{I}, \mathrm{opt}}=T_{1, \text { blood }} \cdot\left[\ln 2-\ln \left(1+\exp \left(-T_{\mathrm{R}} / T_{1, \text { blood }}\right)\right)\right]
$$

The blood $T_{1}$ is long (e.g. $1200 \mathrm{msec}$ at $1.5 \mathrm{~T}$ ) and $T_{\mathrm{I}}$ should be long as well. Moreover, when a $T_{\mathrm{I}}$ longer than $T_{\mathrm{I}, \mathrm{opt}}$ is used the image quality is improved (12), especially for the right ventricle.

In clinical applications, data are generally acquired during the mid-diastolic rest, as for moderate heart rates (50-85 bpm), the beginning of the diastolic rest period is in good agreement with the long inversion time needed to nullify the blood signal (see Fig. 1). In cases of significant RR variations or high heart rates (over $85 \mathrm{bpm}$ ), DIR-FSE is impossible to acquire in mid-diastolic rest (18). In such cases, it is possible to acquire data at end-systole as the end-systolic rest is longer than the mid-diastolic rest and less sensitive to beat-to-beat changes. So in addition to the clinical benefit expected due to the contraction of the myocardium, performing DIR-FSE in end-systolic rest could also be desirable in such cases.

In current clinical applications, the trigger delay $T_{\mathrm{D}}$, defined as the time between the R-wave and the beginning of the acquisition window, is highly constrained by the heart rate and the inversion time. As a consequence, the acquisition is difficult to perform during the end-systolic rest while taking constraints on $T_{\mathrm{I}}$ into account, especially for $T_{2}$-weighting imaging. In addition, if the FSE acquisition is performed in the end systolic rest, the DIR preparation and the acquisition will be performed while the heart is in different positions, leading to a signal loss. To limit this effect, the thickness of the selective inversion, or DIR thickness, is usually made three times larger than the slice thickness.

The basic principle of acquiring data in end-systolic rest, using a DIR-FSE sequence, is to delay the beginning of the sequence to start the DIR preparation before the next Rwave and to place the echo train in end-systolic rest. Two methods are proposed, an adaptive one that uses a Kalman filter for RR interval prediction and a simple one that uses a fixed delay (Fig. 2). The main difference between these two methods is the delay computation technique.

\section{Adaptive Method}

Instantaneous heart rate, which is defined as the time between two consecutive $\mathrm{R}$ waves, also called RR interval, is known to vary from beat to beat. This variation has been demonstrated while breathing, denoted by respiratory sinus arrhythmia (19), but also during breath-hold (20). To model these beat-to-beat cardiac cycle changes, an RR interval modeling has been proposed (21).

Let $S$ be the respiration signal acquired by a respiratory belt and $d S$ its time derivative. Their values at the $n^{\text {th }} \mathrm{R}$ wave time are, respectively, denoted $S_{n}$ and $d S_{n}$. Let 


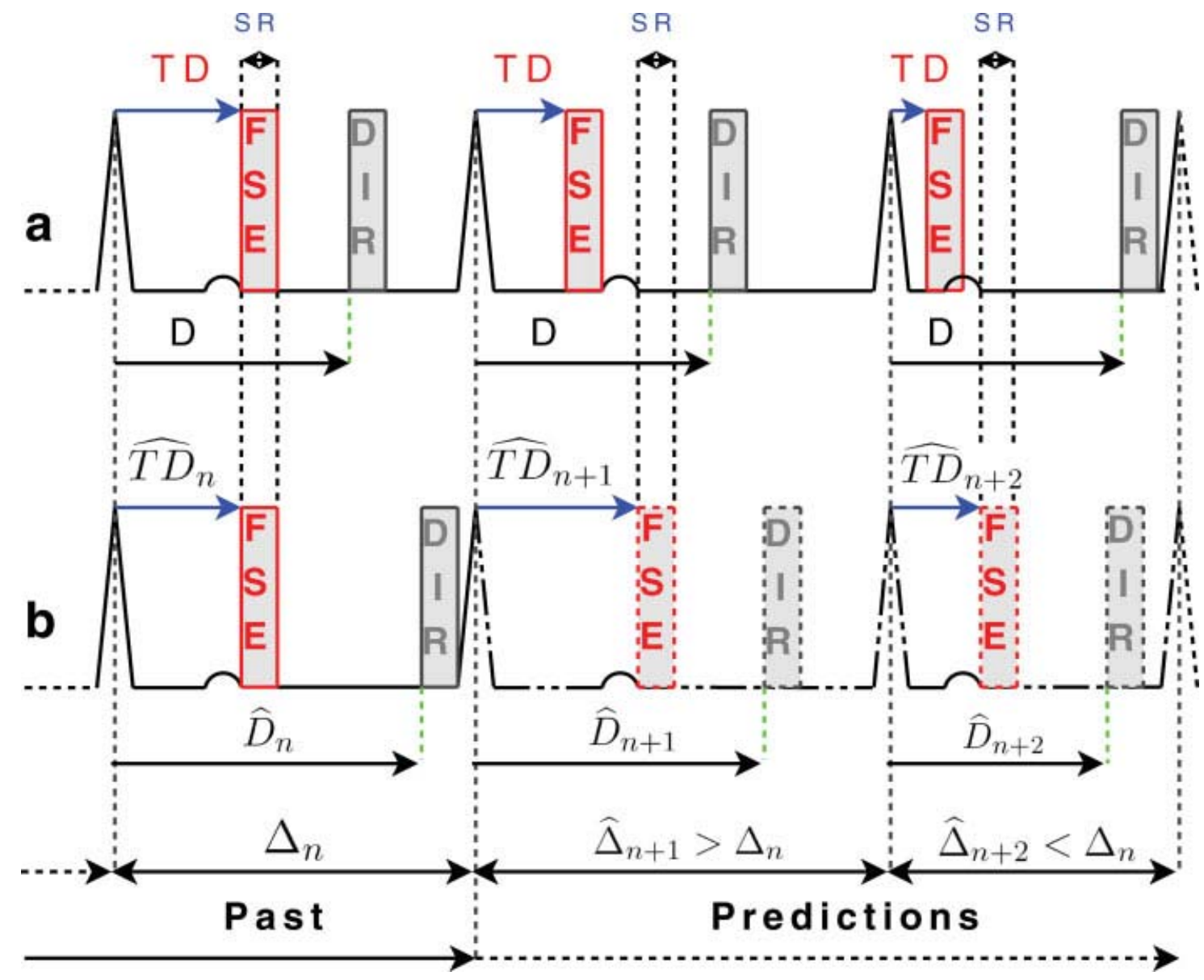

FIG. 2. DIR-FSE pulse sequence in presence of RR interval variations for the fixed fixed delay method (a) and the adaptive method (b). In each cardiac cycle, the end systolic rest is denoted by SR. The inversion time $T_{1}$ is the same for both methods and has been omitted for clarity, $T_{1}$ is the time between the DIR pulses and the FSE sequence. a: Fixed delay method. Based on the average heart rate, sequence parameters and the cardiac cycle model, a fixed delay $(D)$ is computed that enables to place the FSE sequence in end-systole and to keep a long $T_{1}$. b: Adaptive method that uses predictions and the cardiac cycle model.

define $\Delta$ the instantaneous heart rate. $\Delta_{n}$ is defined as the difference between the $n^{\text {th }}$ and $(n-1)^{\text {th }} \mathrm{R}$ wave time. By predicting only one upcoming RR interval $\hat{\Delta}_{n+1}$, the DIRFSE sequence could be started by considering a fixed $T_{D}$ as already proposed but not validated on humans (21). The underlying hypothesis is that the systole has always the same duration. As the data acquisition will be performed in the next cardiac cycle $\Delta_{n+2}$, the variation of the second cycle has to be taken into account because the end-systolic rest is short. Even if the systole duration is less sensitive to RR interval variations, considering only one upcoming RR interval could lead to synchronisation errors. Consequently, a prediction of the two next RR intervals $\hat{\Delta}_{n+1}$ and $\hat{\Delta}_{n+2}$ is used in conjunction with a simple cardiac cycle model to correctly place the acquisition window and to create an adaptive trigger delay. This allows tracking the end-systolic rest from cycle to cycle.

The assumption made in this work is that RR intervals can be modeled as a linear combination of previous RR intervals, respiration and its derivative. Consequently, at the $n^{\text {th }} \mathrm{R}$ wave time, the two next RR intervals are then predicted according to the following equations:

$$
\begin{aligned}
& \Delta_{n+1}=\sum_{i=0}^{p_{1}} a_{i} \Delta_{n-i}+\sum_{i=0}^{p_{2}} b_{i} S_{n-i}+\sum_{i=0}^{p_{3}} c_{i} d S_{n-i}, \\
& \Delta_{n+2}=\sum_{i=0}^{p_{4}} d_{i} \Delta_{n-i}+\sum_{i=0}^{p_{5}} e_{i} S_{n-i}+\sum_{i=0}^{p_{6}} f_{i} d S_{n-i} .
\end{aligned}
$$

The advantage of this modeling is that it uses only widely available signals such as ECG and respiratory belt measurements. This set of equations uses parameters $\left(a_{i}, b_{i}, c_{i}, d_{i}, e_{i}, f_{i}\right)$ that need to be known to accurately predict RR intervals. In this work, a Kalman filter is used to estimate online the set of unknown parameters $\left(a_{i}, b_{i}, c_{i}, d_{i}, e_{i}, f_{i}\right)$ from Eqs. 2 and 3. Kalman filtering $(14,22)$ is a well known and grounded theory and has already been applied to the MR field (23-26). This theory assumes a dynamical state-space model defined by the following pairs of generic equations:

$$
\begin{aligned}
\mathbf{x}_{n+1} & =\mathbf{F}_{n} \mathbf{x}_{n}+\mathbf{u}_{n} \\
\mathbf{y}_{n} & =\mathbf{G}_{n} \mathbf{x}_{n}+\mathbf{v}_{n} .
\end{aligned}
$$


Table 1

Definition of the Constants $A$ and $B$ of the Cardiac Cycle Model

\begin{tabular}{lcc}
\hline & $A$ & $B$ \\
\hline Male & $0.0021 \times 60$ & 0.546 \\
Female & $0.0020 \times 60$ & 0.549 \\
\hline
\end{tabular}

Equation 4, also called process equation, represents the time evolution of the parameter's vector (via the transition matrix $\mathbf{F}_{n}$ ). In the Kalman filter literature, this parameter's vector is also called the state vector. Equation 5 describes the observation process (via the measurement matrix $\mathbf{G}_{n}$ ), that is the relation between observed RR intervals and the parameters. $\mathbf{u}_{n}$ and $\mathbf{v}_{n}$ represent, respectively, the process and measurement noises and describe the uncertainty of the modeling. Further details about Kalman filter are provided in Appendix A.

The linear modeling of RR intervals, Eqs. 2 and 3, is well adapted for Kalman filtering and can be formulated as a dynamical state-space system described by Eq. 6. The evolution of the parameter's vector is modeled by a random walk, as no a priori can be assumed on its time evolution. Thus, the transition matrix $\mathbf{F}_{n}$ is the identity. This modeling can be applied for both free breathing and breath-hold modes, as the Kalman filter aims at adaptively estimating the optimal model parameters $\left(a_{i}, b_{i}, c_{i}, d_{i}, e_{i}, f_{i}\right)$. Using this state-space formulation and the Kalman filtering theory, the prediction of the next RR intervals $\hat{\Delta}_{n+1}$ and $\hat{\Delta}_{n+2}$ can be computed (see Appendix A for details).

$$
\begin{array}{r}
\mathbf{x}_{n}=\left[a_{0}, \ldots, a_{p_{1}}, b_{0}, \ldots b_{p_{2}}, c_{0}, \ldots, c_{p_{3}}, d_{0}, \ldots, d_{p 4},\right. \\
\left.e_{0}, \ldots, e_{p_{5}}, f_{0}, \ldots, f_{p_{6}}\right]^{T},
\end{array}
$$

$\mathbf{F}_{n}=$ Identity,

$\mathbf{y}_{n}=\left[\Delta_{n+1}, \Delta_{n+2}\right]^{T}$

$\mathbf{G}_{n}=\left[\begin{array}{c}\Delta_{n}, \ldots, \Delta_{n-p_{1}}, S_{n}, \ldots, S_{n-p_{2}}, d S_{n}, \ldots, d S_{n-p_{3}}, \\ 0, \ldots, \ldots, \ldots, 0 \\ 0, \ldots, \ldots, 0, \Delta_{n}, \ldots, \Delta_{n-p_{4}}, S_{n}, \ldots, S_{n-p_{5},} \\ d S_{n}, \ldots, d S_{n-p_{6}}\end{array}\right]$

Once the beginning and the duration of the next cycle are estimated, a cardiac cycle model is used to place the acquisition window during the end-systolic rest. This model, which is derived from the description by Weissler et al. (8), gives the systolic and diastolic durations, $T^{\mathrm{s}}$ and $T^{\mathrm{d}}$, respectively, as a function of the RR interval $\Delta$ (Fig. 1a). Note that the systolic duration $T^{\mathrm{s}}$ includes the end-systolic rest. The cardiac model is described as follow:

$$
\left\{\begin{array}{l}
T^{\mathrm{s}}=B-A \cdot \Delta^{-1} \\
T^{\mathrm{d}}=\Delta-T^{\mathrm{s}}
\end{array}\right.
$$

where $T^{\mathrm{s}}, T^{\mathrm{d}}, \Delta$ are expressed in seconds. The constants $A$ and $B$ are defined in Table 1 .

The computation of the Kalman filter at the $n^{\text {th }} \mathrm{R}$ wave time gives the prediction of $\hat{\Delta}_{n+1}$ and $\hat{\Delta}_{n+2}$. Knowing the timing of the DIR-FSE sequence for a given experiment, an optimal trigger delay ${\widehat{T_{D}}}_{n+2}$ and an optimal delay $\hat{D}_{n+1}$ are computed. $\widehat{T}_{D+2}$ corresponds to the time between the $(n+1)^{t h} \mathrm{R}$ wave and the beginning of the FSE echo train, and $\hat{D}_{n+1}$ is the delay between the $n^{\text {th }} \mathrm{R}$ wave and the beginning of the DIR (Figure 2-b). Let define $T_{\mathrm{FSE}}$ as the time between the $\pi / 2$-pulse of the FSE sequence and the end of the echo train. The optimal trigger delay is computed using the twostep prediction, the cardiac cycle model and the duration of the echo train $T_{\mathrm{FSE}}$ :

$$
\begin{aligned}
\hat{T}_{n+2}^{\mathrm{s}} & =B-A \cdot \hat{\Delta}_{n+2}^{-1} \\
\widehat{T}_{D n+2} & =\hat{T}_{n+2}^{\mathrm{s}}-T_{\mathrm{FSE}}
\end{aligned}
$$

Then, the optimal delay $\hat{D}_{n+1}$ is estimated using previous results and sequence timing (e.g. $T_{\mathrm{I}}$ ):

$$
\hat{D}_{n+1}=\hat{\Delta}_{n+1}-T_{\mathrm{I}}+{\widehat{T_{D}}}_{n+2} .
$$

Sequence timing for this adaptive method is represented in Fig. 2b. Note that $T_{\text {FSE }}$ is required for the computation of $\widehat{T}_{D_{n+2}}$ to insure that the complete echo train is included in the end-systolic rest period. This is due to the fact that the systolic duration $T^{\mathrm{s}}$ of the cardiac cycle model includes the end-systolic rest. In this work, the interest of acquiring images in end-systolic rest is demonstrated by using the proposed model in breath-hold. Using the proposed prediction scheme with respiratory signals in breath-hold is still useful as breath-hold are not perfect, particularly in endexpiration. Our hypothesis is that most people make small movements while they are holding their breath, yielding thoracic volume variations that could modify the RR interval variation patterns. Moreover, a previous study (21) has reported some prediction improvements when using respiratory signals in breath-hold.

\section{Fixed Delay Method}

If the heart cycle is considered to remain constant and stable during the whole acquisition, a simple solution to perform DIR-FSE in end-systolic rest exists. It consists in delaying the beginning of the DIR preparation after the last detected R-wave by a fixed delay. For this method, the fixed delay $D$ has been computed using the average of heart rate $\bar{\Delta}$ (average over $9 \mathrm{RR}$ intervals prior acquisition), the sequence parameters, and the cardiac cycle model:

$$
\begin{aligned}
\mathrm{TD} & =B-A \cdot \bar{\Delta}^{-1}-T_{\mathrm{FSE}}, \\
D & =\bar{\Delta}-T_{\mathrm{I}}+\mathrm{TD} .
\end{aligned}
$$

The fixed delay method allows $T_{\text {I }}$ to be kept long by using a fixed delay $D$. Sequence timing for the fixed delay method is represented in Fig. 2a and for the proposed adaptive method in Fig. 2b. Both method charts are illustrated in situation of important RR interval variations.

\section{MATERIALS AND METHODS}

\section{Materials}

MR examinations were performed on a $1.5 \mathrm{~T}$ clinical MR system Signa HDx (General Electric, Milwaukee, WI) with a standard clinical eight elements cardiac coil. The adaptive method, the fixed delay method, and the clinical standard method (2) were implemented using a dedicated homebuilt real-time system independent from the MR scanner called Signal Analyser and Event Controller (SAEC) (27). Commercial ECG sensors with a $20 \mathrm{~Hz}$ bandwidth (Schiller 
Medical, Wissembourg, France) and pneumatic respiratory belt were used to collect physiological signals in the MR bore. A TTL output of the SAEC was plugged in the external triggering input of the MR system. This allowed the gating of the pulse sequence, when needed, with a minimal time response. ECG, respiratory belts, trigger signals and signals from the MR system such as acquisition windows, gradients and RF were recorded by the SAEC to evaluate the reliability of the different methods. As the performance of the proposed adaptive method relies on the quality of the QRS detector, the algorithm used in this work was the same as in an industrial monitoring system (Argus PB-1000, Schiller AG, Baar, Switzerland).

For the adaptive method, a second order RR interval model had been used, meaning that $p_{1}$ to $p_{6}$ are set to 1 in Eqs. 2 and 3. The second order has been chosen because it is a good compromise between accurate prediction and computational efficiency (21) and allows real-time processing. Setting the model to an higher order will improve accuracy of the prediction but lengthen the computation and convergence times. In case of important beat-to-beat changes, slowing the convergence time will increase the time needed to have accurate predictions in subsequent heart cycles. Once an R-wave was detected,an updated set of parameters $\left(a_{i}, b_{i}, c_{i}, d_{i}, e_{i}, f_{i}\right)$ is computed by the Kalman filter on the SAEC and used to estimate the two following RR intervals $\left(\hat{\Delta}_{n+1}\right.$ and $\left.\hat{\Delta}_{n+2}\right)$. The DIR pulses were played after the optimal delay $\hat{D}_{n+1}$ and FSE echo train was acquired $T_{\mathrm{I}}$ msec later, corresponding to the optimal trigger delay of $\widehat{T}_{D_{n+2}}$ msec after the last R-wave. The whole process was repeated until the $k$-space was completely filled. For the implementation of the filter, the state vector $\mathbf{x}_{0}$ was initialized to the previous $\mathrm{RR}$ interval length meaning that $a_{0}$ and $d_{0}$ were set to 1 and other parameters were set to 0 . As the evolution process was simply modeled by a random walk, the evolution noise $\mathbf{u}_{n}$ covariance matrix was set to $\operatorname{diag}(10,10)$ to denote the uncertainty on the state evolution process (Eq. 4). The measurement noise $\mathbf{v}_{n}$ covariance matrix was set to $\operatorname{diag}(0.01,0.01)$ to have a quick convergence of the filter and to denote the confidence on the observation $\mathbf{y}_{n}$. The fixed delay method was implemented by delaying the beginning of the pulse sequence after the last detected R-wave. The delay $D$ was fixed for the whole acquisition. The standard method, which acquire mid-diastolic images, had been implemented as in clinical routine by using the chosen QRS detector. The presented method has not been compared to moving average on five previous RR intervals (28) because an other study (29) has reported improvement by using a predictive method compared to the moving average approach. Moreover, the total examination time has been kept at an acceptable length for the volunteers.

\section{MRI Protocol}

Fourteen healthy volunteers underwent a cardiac MR examination to evaluate and compare the different methods. The studied population was composed of seven males and seven females with an average age of $28.7 \pm 11$ years, an average weight of $70.9 \pm 14.3 \mathrm{~kg}$, and an average body height of $1.72 \pm 0.1 \mathrm{~m}$. This study was approved by the local ethics committee and was conducted in compliance with local laws on clinical research. Informed consent was obtained for all volunteers.

To see both right and left ventricles, a mid-ventricular short axis cine sequence was acquired (balanced steady state free precession bSSFP, $T_{\mathrm{R}}=3.9 \mathrm{msec}$, echo time $T_{\mathrm{E}}=$ $1.7 \mathrm{msec}, \mathrm{FOV}=360 \mathrm{~mm} \times 360 \mathrm{~mm}$, bandwidth $=250 \mathrm{kHz}$, matrix $=224 \times 224$, flip angle $=45^{\circ}$, slice thickness $=$ $8 \mathrm{~mm}$, reconstructed matrix $=512 \times 512$ ) and used as a reference to determine if subsequent acquisitions were effectively acquired in the desired cardiac rest period. The same mid-ventricular short axis view was acquired with the DIR-FSE sequence using the three different methods, depicted on Figs. 1 and 2. The first was the clinical standard method with acquisition in mid-diastolic rest, the second was the fixed delay method, and the third was the adaptive method. The second and the third methods were expected to image the heart in end-systolic rest.

For the clinical standard method and the adaptive method, seven DIR-FSE images, with different $T_{\mathrm{E}}$ ranging from 10 to $70 \mathrm{msec}$, were acquired to get both $T_{1}$ and $T_{2}$ weighting images. For the fixed delay method, from one to seven images, within the same $T_{\mathrm{E}}$ range, were acquired to keep total scan duration at an acceptable length for the volunteers. The other DIR-FSE sequence parameters were set as follow: $T_{\mathrm{I}}=500 \mathrm{msec}$, FOV $=$ $360 \mathrm{~mm} \times 360 \mathrm{~mm}$, bandwidth $=125 \mathrm{kHz}$, matrix $=256 \times$ 256, slice thickness $=6 \mathrm{~mm}$, DIR thickness $=18 \mathrm{~mm}$, echo train length $=16$, reconstructed matrix $=512 \times 512$. These imaging parameters led to an acquisition window of $85 \mathrm{msec}$, which is the maximal acceptable length with regard to the end-systolic rest duration. For all DIR-FSE methods, an extra echo train was played out but not acquired for MR signal stabilization purpose. All sequences were performed in breath-hold of around 16 sec for standard and fixed delay DIR-FSE, $18 \mathrm{sec}$ for the adaptive DIR-FSE, and $12 \mathrm{sec}$ for the cine. For DIR-FSE sequences, an echo train was acquired every RR for heart rate smaller than $85 \mathrm{bpm}$ or every two RR for others. For the adaptive DIR-FSE, volunteers were asked to hold their breath, then the Kalman filter was started. Two or three RR intervals were used to let the Kalman filter converge before starting the sequence.

\section{Data Analysis}

Mean heart rate was retrospectively computed to evaluate if there were significant differences that could disturb results on quantitative measures. The RR variability, defined as the standard deviation of RR intervals, was also quantified for the same purpose. Differences in heart rate and RR variability between acquisitions performed with the fixed delay and the adaptive method were analyzed using the Wilcoxon signed rank test.

\section{Quantitative Assessment}

Two types of quantitative measurements were used to assess the reliability of the adaptive and fixed delay methods. These quantitative measurements consisted in comparing errors made on trigger delays using the adaptive and fixed delay methods with the best achievable trigger delay $T_{\mathrm{D} \text {,best }}$. This $T_{\mathrm{D} \text {,best }}$ was computed retrospectively 
using the recorded ECG, the cardiac cycle model and sequence parameters. The best achievable trigger delay was computed using Eq. 8. The only difference was that RR intervals $\Delta_{n+1}$ and $\Delta_{n+2}$ were real RR intervals extracted from recorded ECG. For both methods, the applied trigger delay $T_{\mathrm{D} \text {,applied }}$ computed in real-time by each method was extracted by using the ECG, trigger and the acquisition window from recorded data. Finally, for the first quantitative measurement, $T_{\mathrm{D} \text {,best }}$ and $T_{\mathrm{D} \text {,applied }}$ were compared in a normalized histogram to quantify the distribution of the absolute error.

The second quantitative measurement consisted of computing the root mean squared error per volunteer defined by :

$$
\text { RMSE }=\sqrt{\frac{1}{N} \sum_{i=1}^{N}\left(T_{\text {D,applied }}(i)-T_{\text {D,best }}(i)\right)^{2}} .
$$

The other data, such as mean heart rate, were expressed as mean \pm standard deviation. Statistical differences between errors made by the fixed delay and the adaptive method were analyzed using the Wilcoxon rank sum test. A $P$-value smaller than 0.05 was considered statistically significant.

\section{Qualitative Assessment}

An image quality comparison was performed by a radiologist with 8 years of experience in cardiac MR examination. DIR-FSE images were displayed in a random order using a clinical visualization tool (Advantage Workstation, General Electric, Milwaukee, WI). In every cases, the observer was blinded to the method used.

First of all, the observer was asked to state if images acquired with the fixed delay and the adaptive method were effectively in end-systolic rest in comparison to the cine images. Then, the observer was asked to determine if images acquired with the fixed delay, the adaptive, and the clinical standard methods are of diagnostic quality or not.

Second, an image comparison was made between images acquired in end-systolic rest with the fixed delay and the adaptive method. Images of the same volunteer with the same $T_{\mathrm{E}}$ were blindly displayed to the observer by pairs. The observer was asked to determine in which image the heart was better depicted or if these images were equivalent. Two features were considered separately: first, the right ventricle wall and trabeculae were considered and second, the left ventricle wall and papillary muscles.

Finally, a second image comparison was made between the best images acquired in end-systolic rest, as previously determined and images acquired in mid-diastolic rest. This comparison aimed at verifying the hypothesis that right ventricle could be better determined in end-systolic rest. The comparison was focused on the right ventricle wall, but the inferior wall and the free wall were compared separately. Once again, the observer was asked to determine in which image these features were better viewed or if they were equivalent.

After the comparison, the radiologist was asked to measure the thickness of the RV inferior and free walls for each volunteer in end-systolic rest and in mid-diastolic rest. This aims to show the thickening of the RV walls during the

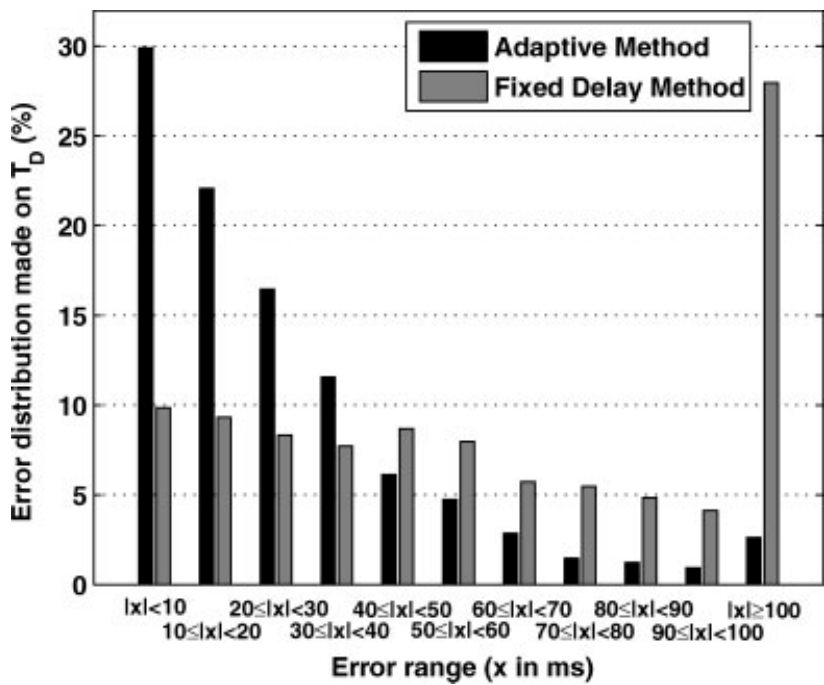

FIG. 3. Normalized histogram of the absolute error made on trigger delay by the adaptive (black) and the fixed delay (gray) methods. This figure shows that error made by the adaptive method are mostly in the small ranges, whereas those made by the fixed delay method are equally distributed. A quarter of errors made by the fixed delay method was over $100 \mathrm{msec}$.

end-systolic rest. The significance of these measurements was assessed with a Wilcoxon signed rank test.

\section{RESULTS}

The MRI protocol was successfully completed on all 14 healthy volunteers. However, one volunteer was excluded from the data analysis due to a faulty ECG sensor, leading to a poor efficiency of the R-wave detector. Therefore, the overall image quality for this volunteer was poor for the three different DIR-FSE methods. The mean heart rate during acquisition in breath-hold of DIR-FSE with the adaptive method was $69 \pm 8 \mathrm{bpm}$ and $68 \pm 9 \mathrm{bpm}$ for the fixed delay method. RR variability was $54 \pm 25 \mathrm{msec}$ for the adaptive method and $49 \pm 22 \mathrm{msec}$ for the fixed delay method. No significant difference in heart rate and RR variability were observed during acquisition performed with the adaptive and the fixed delay method $(P=0.78$ for mean heart rate and $P=0.68$ for RR variability).

\section{Quantitative Assessment}

Figure 3 shows the normalized histogram of the absolute errors made on trigger delay by the adaptive and the fixed delay methods. For example, $80 \%$ of trigger delays estimated by the adaptive method were within a $40 \mathrm{msec}$ error range, whereas only $36 \%$ for the fixed delay method. Moreover, the fixed delay method error was higher than $100 \mathrm{msec}$ in $26.3 \%$ of the cases. This percentage decreased to only $2.6 \%$ for the adaptive method. Figure 3 shows that the adaptive method significantly reduced the number of misplaced acquisition windows compared to the fixed delay method.

The root mean square error, displayed on Fig. 4, was significantly reduced by the adaptive method. The mean and the standard deviation of the error were thus both 


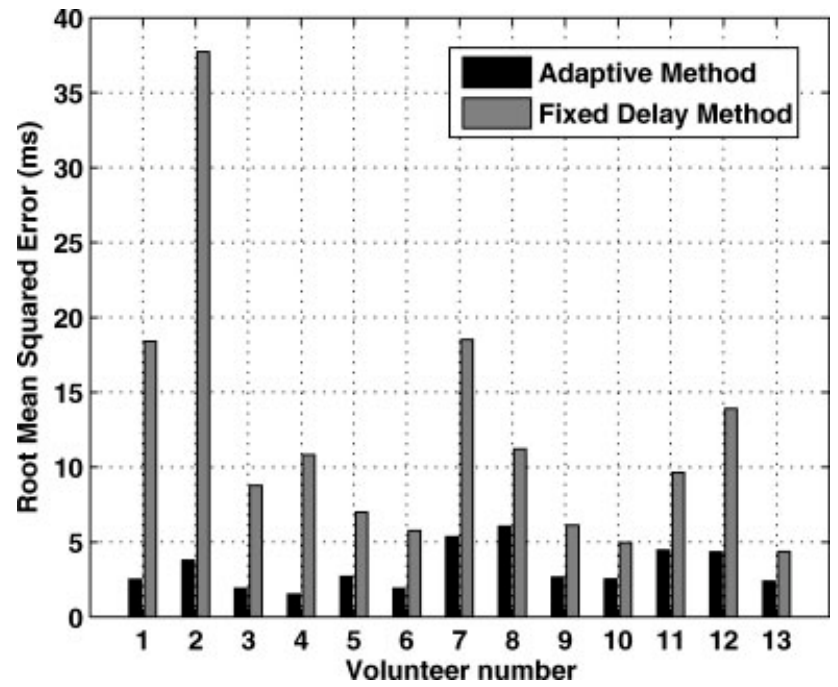

FIG. 4. Root mean square error made in trigger delay between $T_{\mathrm{D} \text {,applied }}$ and $T_{\mathrm{D} \text {, best }}$ for the adaptive (black) and the fixed delay (gray) methods.

reduced by the adaptive method. The overall mean absolute error made was $27 \pm 39 \mathrm{msec}$ for the adaptive method and $75 \pm 98 \mathrm{msec}$ for the fixed delay method with a significant difference $(P<0.001)$.

\section{Qualitative Assessment}

Qualitative results show that $100 \%(n=104)$ of images acquired with the adaptive method are actually in endsystolic rest against $70.6 \%(n=68)$ for images acquired with the fixed delay method. The distribution of the diagnostic image quality is depicted on Fig. 5. This shows that $27.9 \%$ of images acquired with fixed delay method were of nondiagnostic quality; this percentage is reduced to only $1.9 \%$ for the adaptive method and $4.4 \%$ for the standard method.

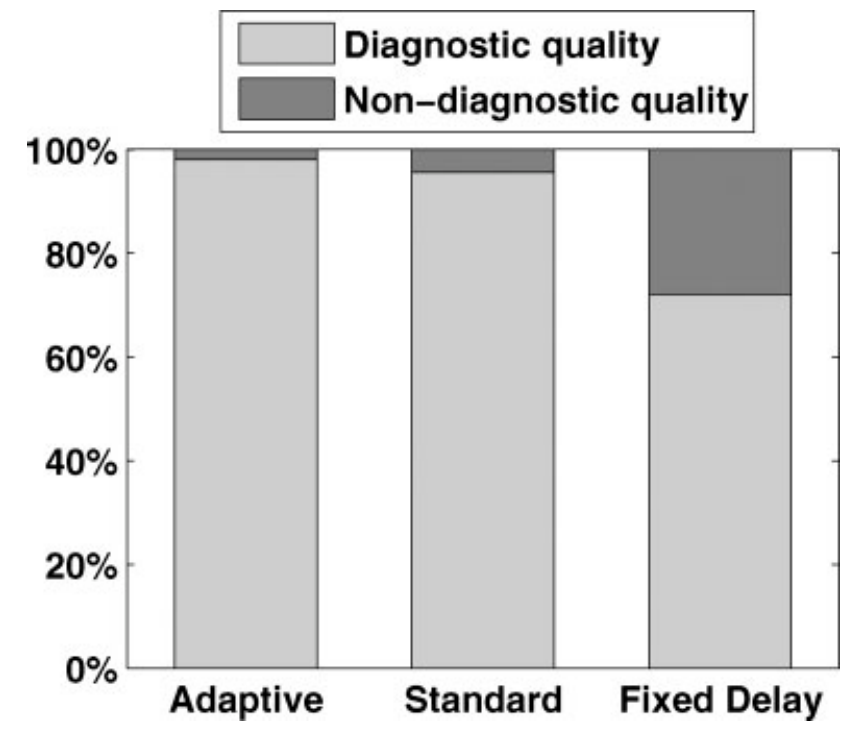

FIG. 5. Overall results on diagnostic quality for the three DIR-FSE methods: the adaptive method $(n=104)$, the clinical standard method in mid-diastolic rest $(n=91)$, and the fixed delay method $(n=68)$.

A total of 68 image pairs acquired with the fixed delay and the adaptive method has been compared by the radiologist. This comparison shows that the right ventricle and trabeculae are better seen with the adaptive method in $65 \%$ of image pairs, with the fixed delay method in $28 \%$, and are equivalent in $7 \%$. The left ventricle and papillary muscles are better depicted in $65 \%$ of image pairs with the adaptive method, $28 \%$ with the fixed delay method, and equivalent in $7 \%$ (Fig. 6).

The comparison of the right ventricle visualization between images acquired in end-systolic rest and in middiastolic rest has been done with all 68 comparable image pairs. The right ventricle inferior wall is better depicted in end-systolic rest in $81 \%$ of image pairs, in mid-diastolic in $15 \%$, and are equivalent in $4 \%$. Among images in

\section{Distrubution of Best Depicted RV and Trabeculas}

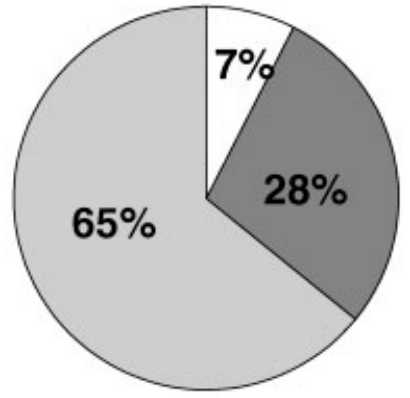

\section{Distribution of Best Depicted \\ LV and Papillary Muscle}

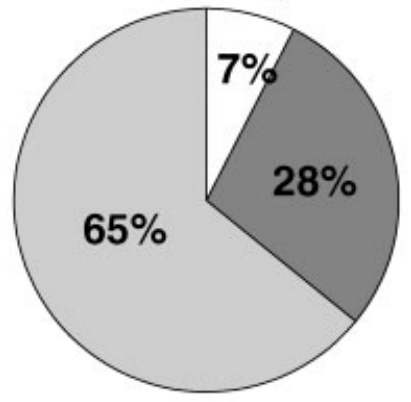

\section{Adaptive $\square$ Fixed Delay $\square$ Equivalent}

FIG. 6. Image quality comparison of the left and right ventricles (LV and RV, respectively) between the adaptive and the fixed delay method. Distribution of methods for which the LV and RV are best depicted. 


\section{Distribution of Best Depicted RV Inferior Wall}

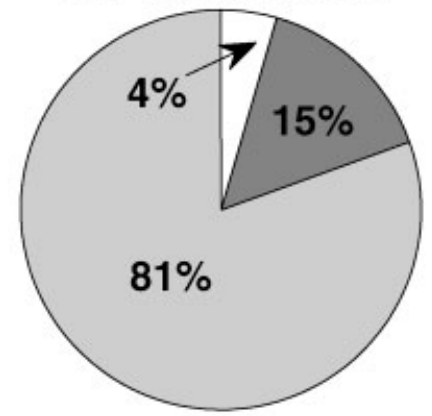

\section{Distribution of Best Depicted RV Free Wall}

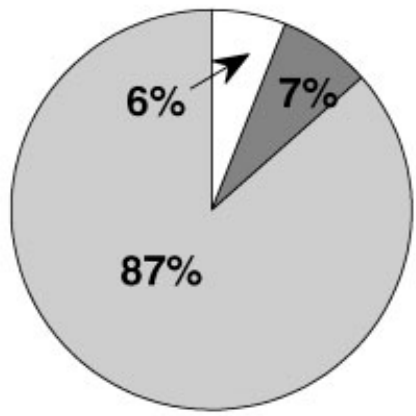

End-Systolic Rest

Mid-Diastolic Rest Equivalent

FIG. 7. Image quality comparison of the right ventricles (RV) inferior and free wall between the mid-diastolic rest and the end-systolic rest. Distribution of rest periods for which the RV inferior and free wall are best depicted.

end-systolic rest, $70 \%$ of images were acquired with the adaptive method. For the right ventricular free wall, $87 \%$ of image pairs are better in end-systolic rest, $7 \%$ are better in mid-diastolic rest, and $6 \%$ are equivalent. Seventy one percent of images in end-systolic rest were acquired with adaptive method (Fig. 7).

The thickness of the RV inferior wall in mid-diastolic rest was $2.53 \pm 0.5 \mathrm{~mm}$ and $4.55 \pm 0.92 \mathrm{~mm}(P<0.01)$ in end-systolic rest. For one patient, the radiologist was not able to measure the RV inferior wall in end-systolic rest. The thickness of the RV free wall in mid-diastolic rest was $2.45 \pm 0.63 \mathrm{~mm}$ and was $4.48 \pm 1.17 \mathrm{~mm}(P<0.01)$ in endsystolic rest. The radiologist was unable to measure the thickness of the RV free wall in mid-diastolic rest on five volunteers due to partial volume effect.

Three images acquired in end-systolic rest with the cine, the fixed delay and the adaptive methods are displayed on Fig. 8. It is worth noting how the right ventricle wall is well depicted with the adaptive method (Fig. 8c), contrary to the image obtained with the fixed delay method (Fig. 8b), which demonstrates the need to take RR interval variations into account for end-systolic rest imaging. To demonstrate the gain in performance black blood FSE in end-systolic rest, images in end-systolic rest and mid-diastolic rest are displayed on Fig. 9. The depiction of the right ventricular free wall is better in end-systolic rest with the adaptive method (Fig. 9c) than in mid-diastolic rest (Fig. 9e).

\section{DISCUSSION}

Imaging the morphology of the heart in end-systolic rest with black blood FSE is not a straightforward task and depends on several patient specific factors. In this work, the feasibility of black blood FSE in end-systolic rest has been demonstrated. The presented method is based on an adaptive RR interval prediction algorithm and an adaptive trigger delay. This method automatically adapts itself to each patient's instantaneous heart rate variation. The adaptive technique was compared to a simple alternative that uses a fixed delay. The results suggest an important improvement for both qualitative and quantitative data. It has been demonstrated that the right ventricle wall is better determined in end-systolic rest. Consequently, the adaptive DIR-FSE should be considered for imaging the right ventricle wall in several clinical cases such as ARVD. A clinical study has to be conducted to demonstrate the

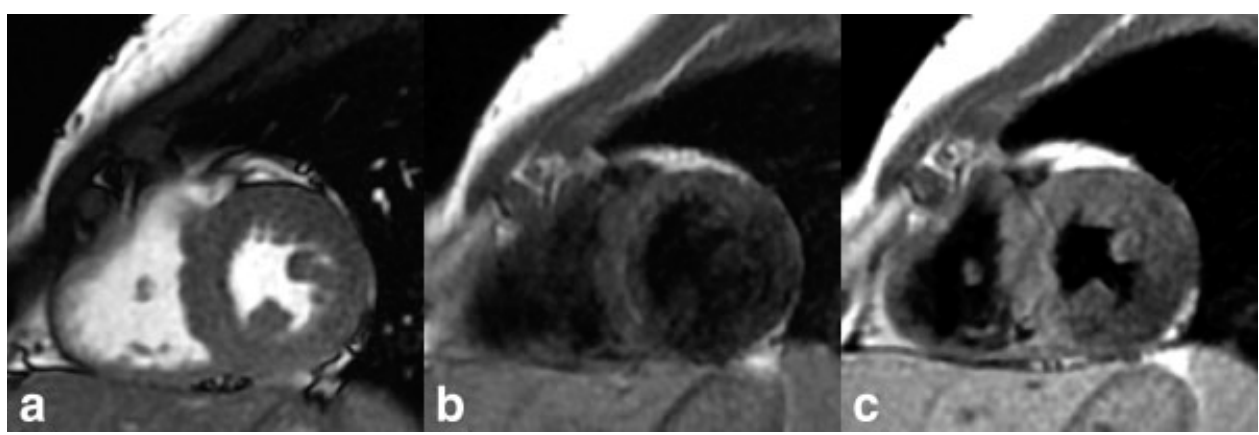

FIG. 8. End-systolic images of volunteer number 12. a: End-systolic image from the cine sequence. b: Image acquired with the fixed delay DIR-FSE method (RMSE $=42.5 \mathrm{msec}$ ) and $\mathbf{c}$ : with the adaptive DIR-FSE method (RMSE $=7.9 \mathrm{msec}$ ). Images (b) and (c) were acquired with $T_{E}=30 \mathrm{msec}$. Notice how the right ventricle free wall is well depicted on (c). 

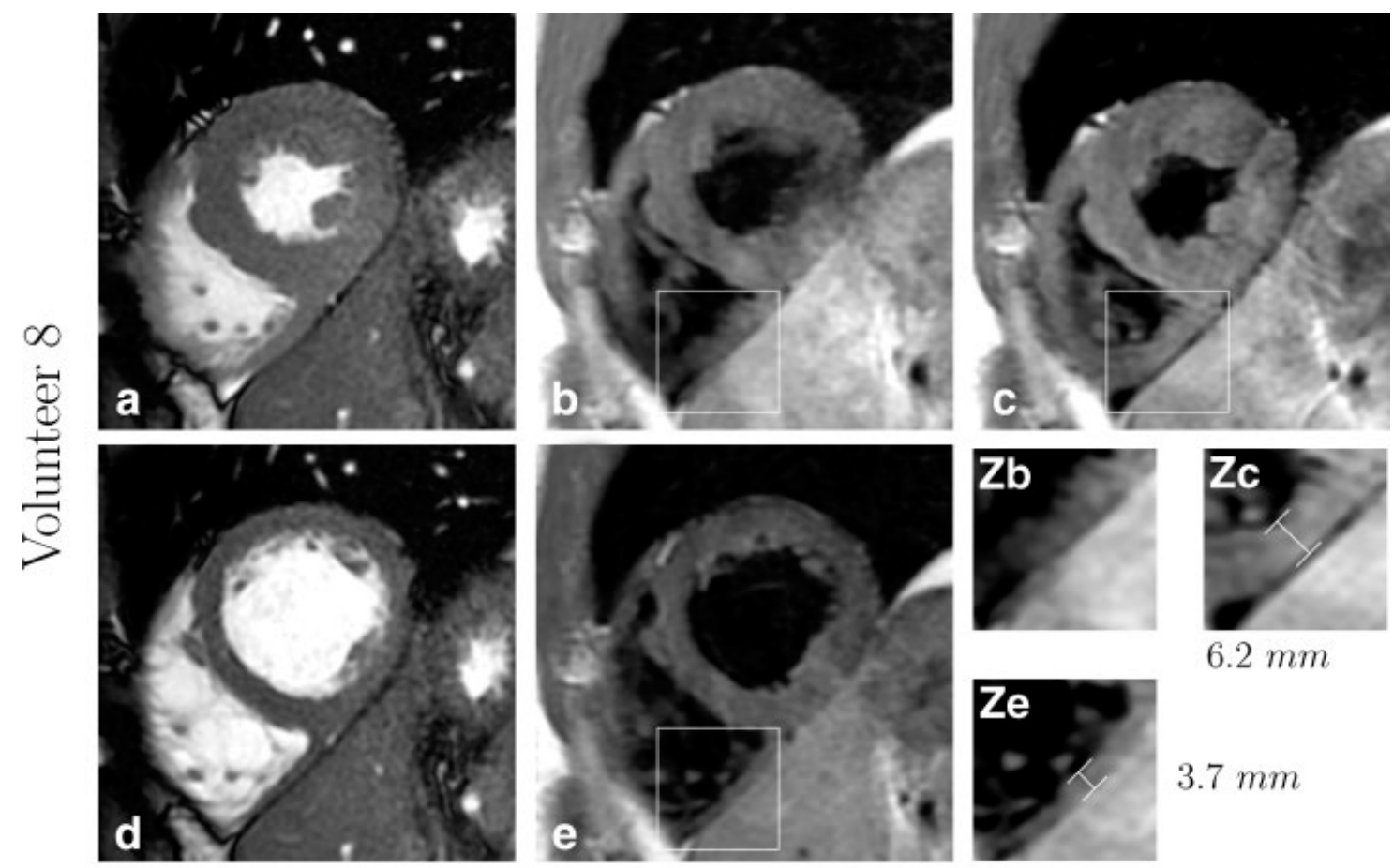

$6.2 \mathrm{~mm}$
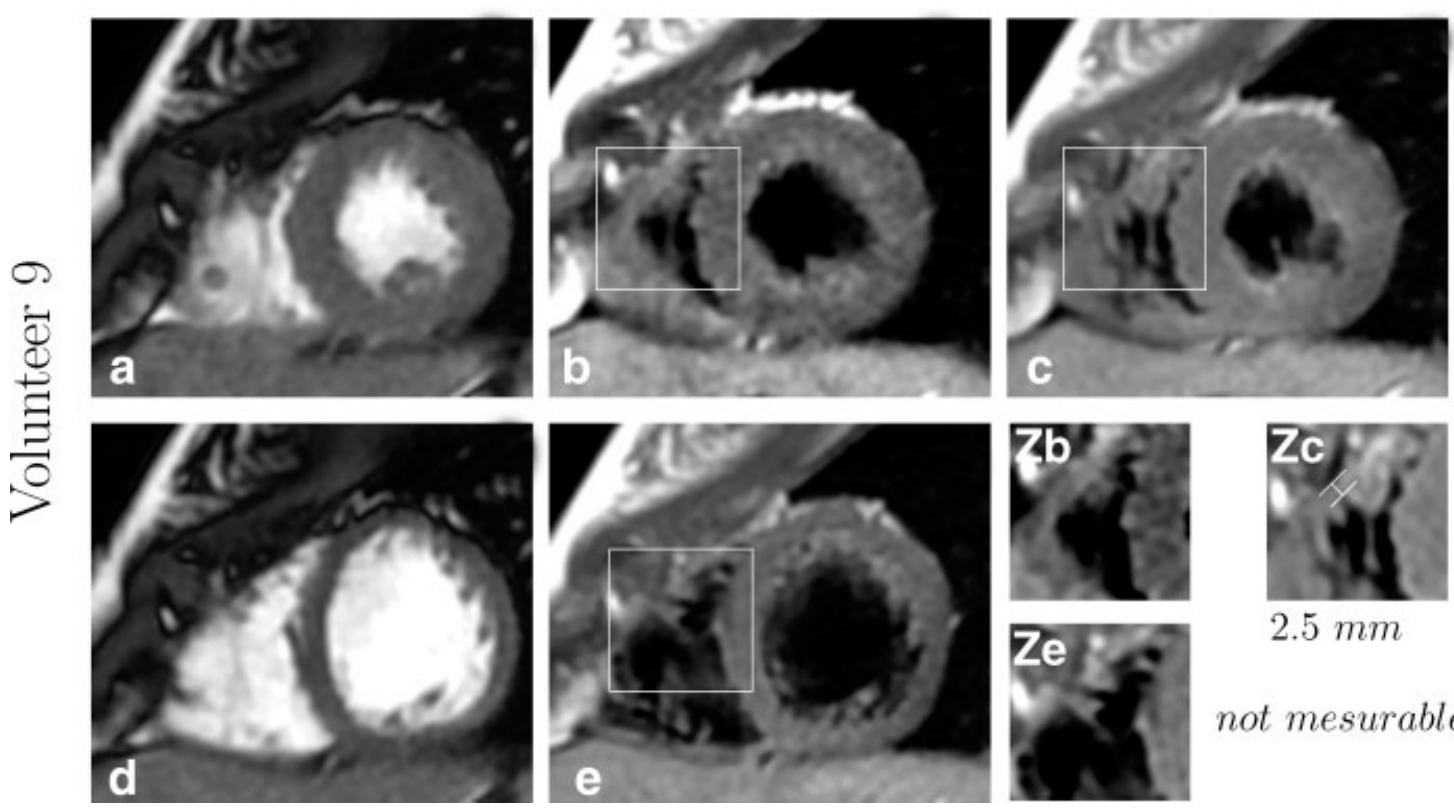

$2.5 \mathrm{~mm}$

not mesurable

FIG. 9. Images of volunteers number 8 and 9. End-systolic (a) and mid-diastolic (d) images from the cine sequence. b: Image acquired with the fixed delay DIR-FSE method (RMSE $=22.4 \mathrm{msec}$ for volunteer 8 and RMSE $=32.5 \mathrm{msec}$ for volunteer 9 ) and (c) with the adaptive method (RMSE $=12.1 \mathrm{msec}$ for volunteer 8 and RMSE $=8.2 \mathrm{msec}$ for volunteer 9 ). e: This was acquired with the clinical standard sequence. b,c,e: These were acquired with $T_{\mathrm{E}}=30 \mathrm{msec}$. Notice how the right ventricle wall and trabeculae are well depicted in end-systolic rest (c) compared to mid-diastolic rest (e). b: These are examples of fixed delay acquisition that leads to suboptimal image quality compared to (c). These images has not been considered to be in end-systolic rest. (Z $\mathbf{Z b}, \mathbf{Z c}, \mathbf{Z e})$ are a zoomed images on the square depicted in images $(\mathbf{b}, \mathbf{c}, \mathbf{e})$, respectively. Right ventricle wall thickness measured by the radiologist is noted when available on $(\mathbf{Z c})$ and $(\mathbf{Z e})$.

usefulness of such methods to diagnose ARVD and other cardiac pathologies. The potential interest of the adaptive method for acquiring data in end-systolic rest has been demonstrated in breath-hold; future work will focus on demonstrating its potential interest in free breathing.

The choice of comparing images by pairs acquired with different methods has been made because this is the simplest solution. As images acquired with different methods are not in the same cardiac rest period, this is a difficult task to grade the same object using a five points scale because, in the present case, the object is in different positions showing different features. This is particularly true on healthy volunteers as there is no particular disease to focus on. This side-by-side comparison allows to reduce 
intra observer variability because answers given by the radiologists are simpler than a grade.

These results are in good agreement with previous studies $(28,30)$, also reporting significant improvement when using an adaptive trigger delay compared to a fixed delay. These methods were designed to improve navigated coronary MR angiography (MRA), which is free breathing acquisition. The adaptive trigger delay was computed to determine the beginning of the mid-diastolic rest and to start the acquisition. These methods are based on a cardiac model and the trigger delay is computed as a function of the RR interval. In these studies, the trigger delay is adapted by using the previous RR interval or an average of previous intervals as a one-step prediction, while in this study, the RR intervals are predicted using a Kalman filter for more accuracy. Coronary MRA is performed in free breathing, so the heart rate variation pattern differs from the one in breath-hold for end-systolic DIR-FSE. This also implies different constraints on the scan time. Note that the prediction algorithm is compatible with free breathing, as respiratory signals are taken into account in the RR interval variation modeling. Consequently, a one step prediction with the adaptive trigger delay could be applied for coronary MRA. Respiratory signals can be acquired with belts and echo navigators. The adaptive acquisition scheme could also be used for the acquisition of end-systolic rest DIR-FSE in free breathing by using a generalized reconstruction including motion correction (31).

The prediction algorithm presented in this work is based on Kalman filtering, which is robust and well known. Other studies has reported a prediction algorithm using a neural network (29) to make an adaptive trigger delay for free breathing coronary MRA. As neural networks are supervised learning models, the training was made using all RR intervals recorded during the navigator preparation phase (around $30 \mathrm{sec}$ ). Therefore, such a method is not directly usable to our specific application. However, it is conceivable to train the model during a breath-hold and then apply the trained model for the acquisition, but this will reduce the efficiency of the examination. Recall that the prediction algorithm converges in two or three RR intervals, which is adapted to acquisitions in breath-hold. This can be seen as if training, and use of the model are performed in a single frame. An interesting alternative solutions to predict RR interval is to use a mix approach of neural network such as a multilayer perceptron and Bayesian or Kalman filtering to online estimate parameters of the perceptron $(32,33)$.

The RR interval linear modeling used in this work has several advantages. Its linear formulation is well adapted to Kalman filtering, holds for everybody and for both free breathing and breath-hold. In addition, the modeling could be more adapted to the physiology of a specific patient thanks to some tuning parameters. This particular point needs further investigations in breath-hold and free breathing on a large population including both healthy volunteers and patients with heart disease.

Several cardiac models have been proposed in the literature, and most of them are based on the QT interval and give its length as a function of the RR interval $(34,35)$. All these models are suitable for end-systolic MR imaging. Additional analysis (34) has shown that some tuning parameters are desirable to adapt cardiac models to the subject being studied depending on age, gender, and the presence of potential heart disease.

To overcome the oversimplified assumption that cardiac cycles are reproducible, a two-step prediction algorithm has been chosen, but numerous alternatives are conceivable. The use of a one-step prediction algorithm and a patient specific cardiac model of the RT interval of the form $\mathrm{RT}_{n+1}=f\left(R R_{n}\right)$ (36) is also an acceptable solution. This model has been initially proposed to adapt the trigger delay for coronary MRA. Unfortunately, this model requires several parameters, such as $\mathrm{RT}_{\max }$, which are usually not available in MR scanners and require an extra ECG recording. This model could be integrated in the Kalman framework as well, with a one-step prediction. The cardiac model used in this work has the advantage of being a simple function of the RR interval but is not patient specific. Consequently, a patient-adapted cardiac cycle model, such as those suggested by Roes et al. (28) and by Liu et al. (36), could potentially be integrated in the Kalman framework. An extra ECG acquisition, inside or outside the MR bore, could be used to compute patients specific parameters.

The short axis view has been chosen to observe both left and right ventricles, as this study was not focused on a specific ventricle. However, a recent study (12) focused on the right ventricle suggests acquiring the mid-ventricular axial view of the heart to get a better visualization of the wall. The proposed method has not yet been tested in the axial plane, but the visualization of the right ventricle could be improved as it is thicker in end-systolic rest. In this work, the same cardiac cycle model has been used for the left and right ventricles with the underlying hypothesis that both ventricles have the same rest periods. As each ventricle could have slightly different rest period, it could be useful to tune a cardiac cycle model for each ventricle to observe ventricles separately in axial view.

A potential problem of the presented method comes from the DIR preparation, which is performed in diastole, whereas data are acquired in end-systolic rest. The heart is thus in two different positions between preparation and acquisition and this could potentially lead to signal loss. To minimize this effect, the DIR thickness has been made three times larger than the slice thickness. Some losses are still noticeable as depicted on Fig. 8c. The DIR thickness was $18 \mathrm{~mm}$ and no specific optimization was made. A larger DIR thickness can remove this problem, but a too large DIR thickness will lead to a partial noncancellation of the blood signal. This particular problem requires further investigations. The motion tracking between preparation and acquisition proposed by Keegan et al. (37) can be an interesting alternative. This tracking method requires the acquisition of two cine sequences with labeling prepulses for the determination of the imaging plane motion throughout the cardiac cycle. These additional acquisitions would lengthen the examination but enhance image quality. Another organ tracking method based on standard physiological sensors could also be used (38), but would also require a calibration step.

No parallel imaging such as Sensitivity Encoding (39) has been used in this study, but this could have various potential benefits. The first one, as suggested by Pruessmann et al. (39), is the acquisition of undersampled $k$-space to reduce scan time or to increase spatial resolution. The 
undersampling could also be used to reduce echo train length, to get it shorter than the systolic rest. The second potential benefit is to retrospectively remove $k$-space lines from a fully sampled data set that were not accurately acquired in the end-systolic rest by using the method described by Odille et al. (40) for cardiac MRI using segmented acquisitions.

The echo train length was set short and the bandwidth large leading to an acquisition window of $85 \mathrm{msec}$, which could still be larger than the end-systolic rest for some volunteers. A fast and accurate automated method to determine the length of the end-systolic rest period could be developed to adapt acquisition parameters to the rest period of each patient.

\section{CONCLUSION}

The results presented in this contribution indicate that the timing of a black blood FSE is a major concern for imaging the morphology of the heart in end-systolic rest. Based on an adaptive prediction algorithm and a cardiac cycle model, a novel method has been described and compared to a simple fixed delay method. Results show the robustness of the adaptive method in terms of trigger delay error and image quality compared to the fixed delay method. A potential interest of end-systolic rest imaging has been demonstrated for the depiction of the right ventricular wall thanks to an image comparison done by an experienced radiologist, and especially for the free wall. End-systolic black blood FSE with the adaptive method should provide additional diagnostic information to that given by the standard black blood FSE in mid-diastolic rest. A large patient population study has to be conducted to demonstrate the diagnostic interest of the proposed method.

\section{ACKNOWLEDGMENTS}

The authors would like to thank the CIC-IT Nancy INSERM CIT801, especially Emmanuelle André for the healthy volunteer recruitment and management, Gabriela Hossu for her useful help in the statistical analysis, Pr. Pierre-Yves Marie, Dr. Valérie Laurent, Dr. Wassila Djaballah from the medical staff for the healthy volunteers medical examinations. The authors also thank MR technicians for their help with volunteers preparation and examination; Schiller Medical for providing part of the instrumentation required; and ASL Europe team for their support, especially Derek Shaw and Patrick Le Roux for their useful comments and corrections.

\section{APPENDIX}

\section{Kalman Filter}

Let consider a linear, time-discrete dynamical system described by the following pair of equations:

$$
\begin{aligned}
\mathbf{x}_{n+1} & =\mathbf{F}_{n} \mathbf{x}_{n}+\mathbf{u}_{n} \\
\mathbf{y}_{n} & =\mathbf{G}_{n} \mathbf{x}_{n}+\mathbf{v}_{n} .
\end{aligned}
$$

The state vector $\mathbf{x}_{n}$, is defined as the sufficient set of variables that describes the dynamics of the system. Typically, the state vector is unknown and then a set of observed data, denoted by $\mathbf{y}_{n}$ the observation vector, is used to estimate it. Equation A1, also called the process equation, represents the evolution of the state vector using the transition matrix $\mathbf{F}_{n}$. Equation A2 describes the observation process with the measurement matrix $\mathbf{G}_{n}$. $\mathbf{u}_{n}$ and $\mathbf{v}_{n}$ represent, respectively, the process and measurement noises and describe the uncertainty of the modeling. They are both Gaussian distributed with zero mean and constant covariance. The covariance of these two noises is generally set empirically depending on the reliability of observed data $\mathbf{y}_{n}$ and on the reliability of process equation.

When $\mathbf{x}_{n}$ is assumed to have a Gaussian distribution, the optimal solution to this problem has been presented by Kalman (14) and is since well-known as Kalman Filter. It consists of an iterative prediction-correction process, in which the sufficient statistics of mean and state-error correlation are calculated and propagated. The recursive computation is composed by the following series of equations:

$$
\begin{aligned}
\mathbf{K}_{n} & =\mathbf{F}_{n} \mathbf{Q}_{n, n-1}^{\mathbf{e}} \mathbf{G}_{n}^{H}\left(\mathbf{G}_{n} \mathbf{Q}_{n, n-1}^{\mathbf{e}} \mathbf{G}_{n}^{H}+\mathbf{Q}_{n}^{\mathbf{v}}\right)^{-1}, \\
\alpha_{n} & =\mathbf{y}_{n}-\mathbf{G}_{n} \hat{\mathbf{x}}_{n \mid y_{1} \ldots y_{n-1}}, \\
\hat{\mathbf{x}}_{n+1 \mid y_{1} \ldots y_{n}} & =\mathbf{F}_{n} \hat{\mathbf{x}}_{n \mid y_{1} . y_{n-1}}+\mathbf{K}_{n} \mathbf{a}_{n}, \\
\mathbf{Q}_{n}^{\mathbf{e}} & =\mathbf{Q}_{n, n-1}^{\mathbf{e}}-\mathbf{F}_{n} \mathbf{K}_{n} \mathbf{G}_{n} \mathbf{Q}_{n, n-1}^{\mathbf{e}}, \\
\mathbf{Q}_{n+1, n}^{\mathbf{e}} & =\mathbf{F}_{n} \mathbf{Q}_{n}^{\mathbf{e}} \mathbf{F}_{n}^{H}+\mathbf{Q}_{n}^{\mathbf{u}},
\end{aligned}
$$

where $\mathbf{Q}_{n}^{\mathbf{u}}$ and $\mathbf{Q}_{n}^{\mathbf{v}}$ are, respectively, the correlation matrix of noises $\mathbf{u}_{n}$ and $\mathbf{v}_{n}$. $\mathbf{Q}_{n, n-1}^{\mathbf{e}}$ is the correlation matrix of the error between $\mathbf{x}_{n}$ and $\hat{\mathbf{x}}_{n \mid y_{1} . . y_{n-1}}$, which is the estimate of $\mathbf{x}_{n}$ knowing observed data $\mathbf{y}$ until time $n-1$. $\mathbf{Q}_{n}^{\mathbf{e}}$ is the correlation matrix of the error between $\mathbf{x}_{n}$ and $\hat{\mathbf{x}}_{n \mid y_{1} \ldots y_{n}} . \mathbf{K}_{n}$ is denoted by Kalman gain and $\alpha_{n}$ by innovation vector. $\hat{\mathbf{x}}_{n+1 \mid y_{1} . y_{n}}$ is the estimate of $\mathbf{x}_{n+1}$ knowing observed data, and is a prediction of the state vector at time $n+1$. Once the prediction of the state vector has been computed, the prediction of the next observed data can be easily computed using the measurement matrix:

$$
\hat{\mathbf{y}}_{n+1 \mid y_{1} \ldots y_{n}}=\mathbf{G}_{n} \hat{\mathbf{x}}_{n+1 \mid y_{1} \ldots y_{n}} .
$$

\section{REFERENCES}

1. Hennig J, Nauerth A, Friedburg H. RARE imaging: a fast imaging method for clinical MR. Magn Reson Med 1986;3:823-833.

2. Simonetti OP, Finn JP, White RD, Laub G, Henry DA. "Black Blood; $T_{2}$-weighted inversion-recovery MR imaging of the heart. Radiology 1996;199:49-57.

3. Abdel-Aty H, Boyé P, Zagrosek A, Wassmuth R, Kumar A, Messroghli D, Bock P, Dietz R, Friedrich MG, Schulz-Menger J. Diagnostic performance of cardiovascular magnetic resonance in patients with suspected acute myocarditis: comparison of different approaches. J Am Coll Cardiol 2005;45:1815-1822.

4. Abdel-Aty H, Zagrosek A, Schulz-Menger J, Taylor AJ, Messroghli D, Kumar A, Gross M, Dietz R, Friedrich MG. Delayed enhancement and T2-weighted cardiovascular magnetic resonance imaging differentiate acute from chronic myocardial infarction. Circulation 2004;109:24112416.

5. Bomma C, Rutberg J, Tandri H, Nasir K, Roguin A, Tichnell C, Rodriguez R, James C, Kasper E, Spevak P, Bluemke DA, Calkins H. Misdiagnosis of arrhythmogenic right ventricular dysplasia/cardiomyopathy. J Cardiovasc Electrophysiol 2004;15:300-306.

6. Bomma C, Dalal D, Tandri H, Prakasa K, Nasir K, Roguin A, Tichnell C, James C, Lima JA, Calkins H, Bluemke DA. Regional differences 
in systolic and diastolic function in arrhythmogenic right ventricular dysplasia/cardiomyopathy using magnetic resonance imaging. Am J of Cardiol 2005;95:1507-1511.

7. Marie PY, Angiö M, Carteaux JP, Escanye JM, Mattei S, Tzvetanov K, Claudon O, Hassan N, Danchin N, Karcher G, Bertrand A, Walker PM, Villemot JP. Detection and prediction of acute heart transplant rejection with the myocardial $T_{2}$ determination provided by a black-blood magnetic resonance imaging sequence. J Am Coll Cardiol 2001;37:825-831.

8. Weissler AM, Harris WS, Schoenfeld CD. Systolic time intervals in heart failure in man. Circulation 1968;37:149-159.

9. Shechter G, Resar JR, McVeigh ER. Rest period duration of the coronary arteries: implications for magnetic resonance coronary angiography. Med Phys 2005;32:255-262.

10. Kim WY, Stuber M, Kissinger KV, Andersen NT, Manning WJ, Botnar RM. Impact of bulk motion on right coronary MR angiography and vessel wall imaging. J Magn Reson Imaging 2001;14:383-390.

11. Jahnke C, Paetsch I, Achenbach S, Schnackenburg B, Gebker R, Fleck E, Nagel E. Coronary MR imaging: breath-hold capability and patterns, coronary artery rest periods, and $\beta$-blocker use. Radiology 2006;239: 71-78.

12. Berkowitz SJ, Macedo R, Malayeri AA, Shea SM, Lorenz CH, Calkins H, Vogel-Claussen J, Tandri H, Bluemke DA. Axial black blood turbo spin echo imaging of the right ventricle. Magn Reson Med 2009;61:307-314.

13. Scott AD, Keegan J, Firmin DN. Motion in cardiovascular MR imaging. Radiology 2009;250:331-351.

14. Kalman RE. A new approach to linear filtering and prediction problems. Trans ASME J Basic Eng 1960;82:35-45.

15. Lee VS. Cardiovascular MRI: physical principles to practical protocols. Lippincott Williams and Wilkins; Philadelphia, PA, USA, 2006. pp 274 282 .

16. Bernstein MA, King KF, Zhou XJ. Handbook of MRI pulse sequences. Elsevier Academic Press; San Diego, CA, USA, 2004. pp 648-659.

17. Fleckenstein JL, Archer BT, Barker BA, Vaughan JT, Parkey RW, Peshock RM. Fast short-tau inversion-recovery MR imaging. Radiology 1991;179:499-504.

18. Kellman P, Aletras AH, Mancini C, McVeigh ER, Arai AE. $T_{2}$-prepared SSFP improves diagnostic confidence in edema imaging in acute myocardial infarction compared to turbo spin echo. Magn Reson Med 2007;57:891-897.

19. Clynes M. Computer analysis of reflex control and organization: respiratory sinus arrhythmia. Science 1960;131:300-302.

20. Raper AJ, Richardson DW, Kontos HA, Patterson JL. Circulatory responses to breath holding in man. J Appl Physiol 1967;22:201-206.

21. Oster J, Pietquin O, Bosser G, Felblinger J. Adaptive RR prediction for cardiac MRI. In Proceedings of the IEEE International Conference on Acoustics, Speech, and Signal Processing, Las Vegas, Nevada, USA, 2008. pp 513-516.

22. Haykin SS. Adaptive Filter Theory. Infornation and system sciences series, 3rd ed., Upper Saddle River, New Jersey 07458: Prentice Hall, Inc.; 1996. pp 302-334.

23. Spincemaille P, Nguyen TD, Prince MR, Wang Y. Kalman filtering for real-time navigator processing. Magn Reson Med 2008;60:158-168.

24. White NS, Shankaranarayanan A, Han ET, Gaddipati A, Roddey C, Dale AM. Propective motion correction using non linear predictive filtering.
In Proceedings of the 15th Annual Meeting of ISMRM. Berlin, Germany, 2007. p 1829

25. Shankaranarayanan A, Han E, Roddey C, White N, Busse R, Kupermanand J, Santos J, Rettmann D, Schmidt E, Dale A. Motion insensitive 3D T2 and T1-weighted imaging with a real-time, image-based prospective motion correction technique (3D PROMO) and automated re-acquisition of motion-corrupted k-space segments. In Proceedings of the 16th Annual Meeting of ISMRM, Toronto, Ontario, Canada, 2008. p 1475 .

26. Sumbul U, Santos JM, Pauly JM. Accelerating dynamic MRI via spatially varying causal windows. In Proceedings of the 17th Annual Meeting of ISMRM. Honolulu, Hawaii, USA, 2009. p 560

27. Odille F, Pasquier C, Abächerli R, Vuissoz PA, Zientara GP, Felblinger J. Noise cancellation signal precessing method and computer system for improved real-time electrocardiogram artifact correction during MRI data acquisition. IEEE Trans Biomed Eng 2007;54:630-640.

28. Roes SD, Korosoglou G, Schar M, Westenberg JJ, van Osch MJ, de Roos A, Stuber M. Correction for heart rate variability during 3D whole heart MR coronary angiography. J Magn Reson Imaging 2008;27:10461053.

29. Buehrer M, Kozerke S, Boesiger P. Trigger delay adaptation during coronary MRA by prediction of heart rate variations. In Proceedings of the 13th Annual Meeting of ISMRM. Miami, Florida, USA, 2005. p 2240.

30. Stuber M, Botnar RM, Danias PG, Kissinger KV, Manning WJ. Submillimeter three-dimensional coronary MR angiography with real-time navigator correction: comparison of navigator locations. Radiology 1999;212:579-587.

31. Odille F, Vuissoz PA, Marie PY, Felblinger J. Generalized reconstruction by inversion of coupled systems (GRICS) applied to free-breathing MRI. Magn Reson Med 2008;60:146-157.

32. Haykin SS. Kalman filtering and neural networks. New York, NY, USA: John Wiley: 2001. pp 23-65.

33. Thodberg HH. A review of bayesian neural netwoks with an application to near infrared spectroscopy. IEEE Trans Neural Netw 1996;7:56-72.

34. Ashman R. The normal duration of the Q-T interval. Am Heart J 1942;23:522-534

35. Malfatto G, Facchini M, Zaza A. Characterization of the non-linear ratedependency of QT intervals in humans. Europace 2003;5:163-170.

36. Liu G, Wright G. Adapting trigger delays to heart rate for coronary MR angiography. In Proceedings of the 14th Annual Meeting of ISMRM, Seattle, Washington, USA, 2006. p 2157.

37. Keegan J, Gatehouse PD, Prasad SK, Firmin DN. Improved turbo spinecho imaging of the heart with motion-tracking. J Magn Reson Imaging 2006;24:563-570.

38. Fernandez B, Lohezic M, Vuissoz PA, Felblinger J. New approach for affine transform parameters estimation for prospective motion correction using external sensors. In Proceedings of the 16th Annual Meeting of ISMRM, Toronto, Ontario, Canada, 2008. p 3118.

39. Pruessmann KP, Weiger M, Scheidegger MB, Boesiger P. SENSE: Sensitivity encoding for fast MRI. Magn Reson Med 1999;42:952-962.

40. Odille F, Vuissoz PA, Pasquier C, Fernandez B, Felblinger J. Motion artifact correction in cardiac MRI as an irregularly sampled imaging reconstruction. In Proceedings of the 15th Annual Meeting of ISMRM, Berlin, Germany, 2007. p 872. 